\title{
La resistenza contro i trend tecnologici che colonizzano la creazione artistica. Conversazione con Giacomo Verde Roma, Hackmeeting, aprile 2006
}

\author{
A cura di Mariano Equizzi
}

\begin{abstract}
Utente-creatore /hardware+software /carrier-istributore Un ordinato sistema i triangoli contigui.

Ora. Totale noise-caos fra creatori/fruitori/distributori. L'orizzonte in cui dovrebbero muoversi i produttori, ma si muovono già i fruitori maggiormente consapevoli di questo Digidrome.
\end{abstract}

Mariano Equizzi

“...Oggi invece l'immaginario viene creato in tempo reale e secondo me appartiene sempre al presente. Quindi l'arte per me non cambia il mondo, semmai è il mondo che cambia l'arte. L'arte non può far altro che cercare di stare al passo con i tempi. Mi piange il cuore nel dire questo, perché io ho iniziato a fare arte pensando di poter cambiare il mondo. Ma non è vero niente."

Questo è un estratto di una intervista che hai concesso a Tatiana Bazzichelli qualche anno fa, e credo che sia il punto di partenza di questa conversazione.

Vedo che non solo l'arte segue il mondo, ma gli stessi artisti sono travolti dai cambiamenti delle merci del mondo. Oggi non c'è pratica artistica che non sia merce da vendere sin dal suo momento progettuale: software da comprare, telecamera da cercare al prezzo più basso negli store ecc ecc. Insomma tutto il sistema della produzione artistica digitale è di fatto un invito all'acquisto di beni tecnologici "aggiornati, e spesso senza questi non ci sono spazi in festival importanti. Mi sembra che siamo di fronte al caos di cui parlava Gibson nei suoi libri circa la difficoltà di trovare un dispositivo ONO-Sendai ad un prezzo vantaggioso, avverto molto tecnofeticismo (persino il telegiornale sarà trasmesso in HD!!!).

E questa corsa al prezzo giusto e al software giusto non mi pare che possa dirsi un trend positivo all'interno del DIGIDROME.

L'impero delle merci cerca di connettere a sé stesso le arti, anche quelle più arcaiche (Teatro di strada, performance, azione immediata, giocolieri e danzatori) e questo link non genera creativi ma utilizzatori, tecnofeticisti dipendenti. 


\section{Vorresti tratteggiare la tua esperienza in modo da stressare su punti importanti come}

la lotta contro il feticismo tecnologico?

Ti rispondo utilizzando il software AbiWord della distribuzione Linux Dyne:Bolic di Jaromil. Tu mi hai mandato le domande in un file PDF che ho scaricato sul PC di casa (ho ancora WIN98Se, presto passerò a Linux). L'ho subito "convertito" in formato TXT, molto più leggero, e I'ho copiato sulla "penna USB" da 256MB per trasferirlo sul PC portatile: un "vecchio" Amilo Fujitsu Siemens del 2002. Siamo ad aprile del 2006 e come puoi vedere le "macchine" che uso non sono certo di ultima generazione. Prima di comprarmi una nuova "cosa" aspetto sempre di averne davvero bisogno. Fin da quando ho iniziato a fare video nell'83. La prima telecamera con VHS portatile I'ho noleggiata alla TeleRent (dava la possibilità di comprare le macchine dopo un periodo di noleggio), anche il Tvcolor e il VHS da tavolo li avevo noleggiati. Con questa attrezzatura ho realizzato i primi video. Il primo riuscito è stato Fine Fine Millennio. Avevo registrato diverse immagini di guerra dalla televisione. Le rimandai sul Tv di casa e le ripresi scarabocchiando lo schermo con pastelli a olio e chine colorate. II montaggio lo feci in macchina. II sonoro con l'audio-dub (ti ricordi cos'è?) del VHS collegato al microfono della telecamera che "ascoltava" le musiche trasmesse nella stanza da due mangianastri, un lettore VHS, una radio e una tastierina mini-casio. In quella prima versione il video si chiamava Di Collegno, il tema era il rapporto tv-guerra-memoria, ed era dedicato al famoso fatto di cronaca dello smemorato di Collegno. Mi ricordo che non lo mandai al Festival U-Tape di Ferrara perché pensavo che siccome era fatto in VHS non lo avrebbero mai accettato. E sbagliai. Ancora non avevo la coscienza "politica" di quello che avevo fatto. Fu quando lo trasformai in Fine Fine Millennio che cominciai a capire il valore di quel lavoro. Ormai era il 1987. Avevo iniziato a collaborare con uno studio di produzione (BlowUp audiovisivi di Treviso di Gabriele Coassin), facevo le riprese ai matrimoni e l'aiuto nei documentari. Nei tempi morti dello studio potevo usare le centraline di montaggio e i mixer video per provare a fare "robe creative". Potevo usare il formato U-matic (quello professionale con le cassette grandi). Mi resi conto che il tipo di "elaborazione" che avevo ottenuto scarabocchiando e riprendendo lo schermo era di una qualità più interessante di quella ottenuta dai sofisticati mixer video. Così remixai Di Collegno passandolo su U-matic (ma senza toccare 
il montaggio) e aggiungendo solo nella seconda parte (quella più astratta) gli effetti di alterazione cromatica e di "motion" offerti dal mixer elettronico. Inoltre cambiai la colonna sonora: il sesto movimento della Patetica di Chaikowsky al posto della sonorizzazione selvaggia della prima versione: un bel "contrasto armonico" tra immagine e suono.

Stavolta lo inviai a diverse rassegne rivendicando il fatto che la matrice era di "bassa tecnologia" (anche se remixato in U-matic, che comunque non era più lo standard più "figo") e mettendo l'accento sulla necessità di una ricerca linguistica che andasse oltre il fascino delle ultime tecnologie proposte dal mercato.

Fine Fine Millennio ebbe un buon successo. E ancora devo dire che "funziona".

Ricordo che i colleghi della BlowUp erano sempre sorpresi nel constatare come riuscissi ad avere dei buoni risultati montando in macchina e girando con un VHS. Questo accadeva tra la fine degli anni ‘ 80 e i primi ‘90, ero riuscito a comprarmi una videocamera e a realizzare miei video.

Riuscii anche a vendere alla RAI delle riprese in VHS fatte in Senegal nel '90, durante un viaggio di scambio culturale con il Teatro delle Albe.

Sempre in quel periodo (1989) mi capita di "inventare" il Tele-racconto. Se non avessi avuto una attenzione particolare all'utilizzo creativo delle "attrezzature domestiche" certo non mi sarebbe capitato di inventare questa fortunata "tecnica" di narrazione teatrale con il video.

\section{Ma cos'è il Tele-racconto?}

"Una telecamera riprende piccole storie di "oggetti", animate da un narratore in tempo reale e ben visibile dagli spettatori. Un televisore le ritrasmette in diretta, come se fosse una potente lente di ingrandimento, ingigantendo le piccole azioni fino a dargli un senso estetico e narrativo altrimenti non percepibile. Così nasce il Tele-racconto.

Attraverso la ripresa in "macro" i piccoli oggetti diventano personaggi e lo spazio di un foglio di quaderno un set televisivo dalle (quasi) infinite possibilità».

Questa è la scheda di presentazione ancora in uso. Certo è che il T-R è più facile da fare che da descrivere. Da quella prima esperienza dell'89 ad oggi la tecnica si è evoluta fino a mescolarsi con i programmi di Vjing. Ma è rimasta sempre costante l'intenzione di 
"smitizzare" le macchine e di creare una tecnica facilmente utilizzabile anche da chi non ha particolare dimestichezza con la tecnologia.

La questione rimane sempre quella che ho detto a Lello Voce quando mi intervistò per Kult:- lo appartengo ad una classe sociale "medio-povera": ho scelto di non avere un lavoro fisso e garantito ma di fare quello che mi piaceva: di divertirmi facendo lavori artistici socialmente utili. In questo modo non mi arricchisco, proprio come la maggior parte della gente che invece ha dovuto scegliere un lavoro normale da salariato, ormai comunque precario. Quindi mi trovo nella stessa condizione della "classe lavoratrice" e di tutti quelli che non possono decidere direttamente il futuro economico e sociale del mondo. Noi non possiamo permetterci di usare grosse tecnologie per esprimere le nostre idee e il nostro immaginario. Le grosse tecnologie le usa l'industria culturale del cinema, della TV, dei grossi musei, della pubblicità, dei produttori di computer o di connessione. Ci fanno credere che loro hanno la delega ad esprimere i nostri sogni e i nostri desideri perché sono più creativi e perché hanno successo. Ma questo è falso perché il successo ormai si crea (basta avere soldi da investirci o saper giocare con i media) e soprattutto perché il vero scopo di tutti quelli che lavorano nell'industria culturale è di arricchirsi (per chi gestisce il denaro) o conservare il proprio posto di lavoro (per chi presta la propria opera) accettando e propagandando la logica del profitto attraverso la separazione tra produttori=creativi e spettatori=passivi. Questo è ancora più vero con la mitizzazione dell'interattività attualmente in corso: si spaccia per "opzione interattiva" il fatto di poter scegliere tra diverse proposte, piuttosto che dare la possibilità di crearne di proprie. Se tutti i soldi e le energie che vengono spesi per creare i prodotti-oggetti culturali venissero spesi per alfabetizzare, distribuire conoscenze tecnico-linguistiche, creare strumenti e opere di relazione tra persone... la vita sul pianeta sarebbe senz'altro migliore. Ma cosa possiamo aspettarci da governi che continuano ad investire migliaia di miliardi in armamenti quando ne basterebbero molti meno per eliminare la fame nel mondo e diffondere una cultura della pace? Comunque io continuo a stare dalla parte dei "subalterni" e dei disubbidienti: così come mi sono rifiutato di fare il militare continuo a cercare di fare oper'azioni, piuttosto che oggetti, che inventino tecniche e possibilità di 
espressione anche per chi non è considerato creativo e dispone di pochi soldi. E questo modo di pensare mi è stato utile anche quando ho usato hi-tech.

La tua Minimal TV e le esperienze di teatro in Kit sono non solo delle interessanti sperimentazioni ma esprimono soprattutto una chiara volontà nel senso della collettivizzazione dell'arte tecnologica e dei processi tecnologici connessi con lo spettacolo contemporaneo. Questo orientamento didattico ha secondo me un valore ben più grande dello show propriamente detto.

Certamente. Per me il processo è importante quanto il risultato. È stupido utilizzare delle modalità autoritarie, o piramidali, per creare un'opera che parla di libertà, o che semplicemente esprime creatività. Ma purtroppo questo avviene ancora nella maggioranza delle creazioni artistiche. Se non si cambiano le modalità produttive i risultati rimangono nella sostanza simili, anche se apparentemente diversi tra loro. Se non si creano le condizioni che permettono di superare alla radice la divisione tra "professionisti dell'attività creativa" e "professionisti della passività creativa" (questo è il nuovo ruolo che viene chiesto allo spettatore) ci sarà sempre una maggioranza costretta a subire "allegramente" le creazioni di una minoranza. Anche in questo l'arte è semplicemente lo specchio del mondo in cui viviamo: dei suoi rapporti di potere.

Condividere il sapere creativo è "fare opera". È un'opera immateriale, che non si può mostrare, ma certamente regala più emozioni ed esperienze cognitive della grande maggioranza delle "opere d'arte", su qualsiasi supporto esse vengano mostrate. Questa visione si scontra apertamente con il "mercato dell'oggetto artistico" perché una lezione, un incontro didattico, non può essere duplicato e rivenduto con il relativo plusvalore. Questo avviene quando si confonde "I'esperienza" con "l'informazione". Ovvero quando si rivende la registrazione di una conferenza o di un incontro: ma la registrazione non avrà mai la stessa potenza dell'incontro.

Nel caso della MinimalTv si trattava di mettere davvero la Televisione nelle mani della gente. La didattica consiste nel far sperimentare cosa è una diretta tv e come cambia la percezione di sé stessi e dei propri conoscenti, del proprio ambiente. È il primo passo per rendersi conto dello scarto tra "l'immagine Tv" e le situazioni riprese. Ma con gioco e diverti- 
mento, senza pedanterie e utilizzando le attrezzature che tutti possiamo avere in casa. II messaggio ancora una volta è quello di smitizzare il valore dei media e dei suoi "professionisti". Tutti possono fare televisione. Così come tutti possono fare teatro se gli vengono forniti gli strumenti necessari. Ed ecco la creazione collettiva di un Kit Drammaturgico per non professionisti. Chiaramente l'elaborazione di un strumento del genere presuppone una idea di teatro che spesso contrasta, ancora una volta, con quella espressa dai teatranti ecc. ecc...

Il tuo lavoro si pone come un chiaro processo di demistificazione del sistema dei media e dei processi industriali che questo sistema crea come gabbie non solo del lavoro cognitivo ma anche della pratica di palcoscenico, pratica vecchia quanto l'uomo. Questa tua tendenza verso quale principale obiettivo porta?

Questa mia tendenza si nutre della convinzione che la creatività deve essere a disposizione di tutti. Che il sapere e l'arte non devono essere fonte o frutti di privilegi. Nessuno deve lucrare sul mio immaginario!!! L'immaginario collettivo non deve essere gestito solo dai professionisti di turno ma deve essere veramente frutto della collettività. Solo così potrà esprimere veramente le paure, i sogni, le speranze, i problemi delle diverse componenti sociali e umane. La tendenza che spingo è la dissoluzione dell'arte e degli artisti, ma non verso un abbassamento del livello qualitativo (come temono e auspicano gli specialisti) ma verso una immensa consapevolezza e creatività diffusa, che metta definitivamente fuori gioco i parametri estetici dominanti. Oggi il giusto ruolo di ogni professionista della creatività è fare in modo che non ci sia più bisogno di lui. Ogni artista deve lottare per la propria liberazione. Per liberarsi dal dovere di essere creativo al posto di chi è costretto a non esserlo perché deve "lavorare ubbidendo". Oggi l'artista è alienato come il non-artista. Liberare gli artisti fa parte di una strategia che intende liberare tutti gli esseri umani dalla schiavitù del lavoro alienato.

L'attività didattica che porti avanti che figura vuole creare, o meglio quali muri vuole abbattere nelle teste di chi partecipa ai tuoi workshop?

Cerca di abbattere tutte le separazioni di genere artistico, di rispetto aprioristico degli esperti. Cerca di non dare per scontato quello che viene suggerito nei "libretti di 
istruzione". Mi interessa evidenziare come i contesti cambiano il senso delle cose. E che se ti vuoi occupare di un aspetto specifico devi cercare di conoscere il più possibile quello che c'è prima, dopo e attorno. Cerco di mostrare che si possono ottenere risultati belli o interessanti seguendo strade alternative e anche con pochi mezzi. E che la creazione artistica è più facile di quanto si pensi.

Permettimi una volgare provocazione (che come tutte le volgari provocazioni hanno uno scopo metaforico), semmai tu fossi insegnante di recitazione e messa in scena nella scuola della De Filippi che cosa diresti il primo giorno ai tuoi allievi?

Sono certo che non succederà mai per questo rispondo volentieri. Direi... lo non sopporto gli "attori", ovvero non sopporto quelli che aspettano e sperano di lavorare con qualche grande (?) regista... non sopporto quelli che mettono il valore del proprio mestiere nelle mani di un altro. Quelli che aspettano di "essere chiamati" o superare un provino per esprimere sé stessi. Fare l'attore (fingere in scena) è la cosa più facile e stupida che esista: basta volerlo fare veramente. Tanto poi in questo sistema dello spettacolo il lavoro non si trova perché sei bravo ma solo perché sei un "amico". Forse anche per questo la trasmissione della De Filippi si chiama Amici.

lo preferisco i "teatranti" ovvero quelli che decidono di fare il proprio spettacolo superando le divisioni di ruolo tra regista, attore, scenografo ecc. ecc. Che discutono, si accapigliano, trovano il modo di accordarsi senza scontentare nessuno, che si scambiano i ruoli e si aiutano a vicenda. È più faticoso ma certamente più bello. Quindi penso che abbiano sbagliato ad invitarmi in questa trasmissione e do immediatamente le dimissioni.

Siamo entrambi appassionati di Science Fiction, non credi che Videdrome sia un Minimal TV? (celebre azione tecno-minimale di Quinta Parete Network). La visione di Cronemberg è del tutto simile a quella tua, ovviamente non hai mai torturato nessuno, ma la volontà di penetrare nei meccanismi oltre la facciata (nel caso di Videodrome era il porno) credo che sia comune con il maestro canadese.

Certo l'intenzione è la stessa anche se le modalità e risultati sono diversi. Cronemberg racconta mentre io anzi noi, perché la MinimalTv non poteva esistere se non c'era- 
no anche gli altri di Quinta Parete Network, facciamo provare direttamente. Cronemberg usa il cinema e alla fine rimane comunque incastrato in quel sistema, io uso tecnologia domestica e sono davvero ai margini del sistema dei media.

È comune nella tua dimensione di ricerca il termine contagio. Vorresti spiegarci la necessità di questo termine.

La cultura si diffonde per contagio. Le idee e i comportamenti si diffondono e si sviluppano per contagio. Ovvero attraverso il contatto e la trasmissione da una persona all'altra. Nel sistema della comunicazione di massa si assiste ad una sorta di "infezione collettiva" che ha bisogno di essere continuamente alimentata. Oppure, al contrario, si può dire che si assiste ad una sorta di "disinfestazione di massa" che continuamente cerca di eliminare i contagi nati dal contatto tra persone, o di favorirne solo lo sviluppo di alcuni. Per me il contagio è la tecnica di diffusione del sapere più potente che esista, perché utilizza modalità "biologiche" che sono più antiche e profonde di quelle "meccaniche".

Credo, studiando i tuoi materiali presenti in rete e il tuo sito (http://www.verdegiac.org), che tu cerchi di creare strumenti ed azioni per distruggere paradigmi, quelli che ti hanno fatto dire in quella intervista con T Bazz quella frase così dura e che condivido pienamente.

Quello che so è che i paradigmi che hanno cercato di insegnarmi a scuola, in chiesa o in famiglia non corrispondevano al mondo in cui vivo. La discrepanza tra il dire e il fare è una delle cose che mi ha sempre colpito. Sento dire cose e poi ne vedo altre... quello che cerco di fare e creare barche che mi aiutino a superare il mare che spesso trovo tra il dire e il fare. Per fare barche serve materiale galleggiante: i pezzi dei falsi paradigmi mi sembrano molto adatti.

Non pensi che sia una eccessiva tendenza da parte delle arti tecnologiche a gestire sempre maggiori strumenti "difficili" come MAX-Msp-Jitter? E questo tipo di tipo di tools così evoluti e anche criptici non pensi che possano creare, preparare degli artisti asserragliati in alti castelli tecnologici impenetrabili a qualunque coscienza comune? 
Siamo ancora nell'ambito della "mitizzazione" delle macchine, oppure dell'ultimo strabiliante software. È vero che il tempo speso per imparare a gestire "I'ultima novità" è tempo sottratto al sociale. Ma questo avviene anche per i pittori che continuano ad usare il pennello nel chiuso dei propri studi. Ancora una volta la colpa non è delle macchine ma delle persone che le usano. Se non si vive nella "fretta" o con l'ansia del risultato si può benissimo usare software complessi e trovare il tempo per occuparsi del mondo che ci circonda.

Se dovessi gestire un teatro o uno spazio polivalente, quali sistemi hardware e software impianteresti, immagino che anni di tournée ovunque e in spazi alternativi ti abbiano creato nella mente un luogo immaginario dove potere lavorare senza troppi problemi. Chi si occupa di "nuove" cose nell'ambito dell'arte performativa soffre un continuo stato di insufficienza e carenza, logistica, tempistica e tecnologica molte volte legata al luogo in cui la performance dovrebbe attuarsi. Non credi che lo stesso teatro come luogo dovrebbe cambiare in una direzione che assecondi le azioni e i cambiamenti dei lavori presentati, un luogo che rispetti i nuovi artisti che sono nati in questi anni?

È vero, il teatro dovrebbe essere quello che dici tu. Ma purtroppo non lo è. Anzi in questo momento, specialmente in Italia, è quanto di più obsoleto e chiuso possa esistere. Infatti ho deciso, per la seconda volta nella mia vita, di abbandonare il teatro. Ho provato in questi ultimi sette anni a rientrare "nel giro". Avevo smesso nei primi anni '90 per ricominciare nel 1999. Ma purtroppo la situazione dei teatranti italiani è veramente disastrosa. Salvo rare eccezioni. Hanno perso quasi completamente il rapporto con gli spettatori, con i propri territori. Si preoccupano di inseguire delle estetiche e delle problematiche espressive decisamente "autoreferenziali". II mercato e la distribuzione degli spettacoli sono gestiti da logiche che sfiorano il sistema mafioso. Vedono con molta diffidenza l'utilizzo creativo delle tecnologie video o digitali, non ne capiscono le potenzialità espressive, e le usano quasi sempre per darsi solo una veste di modernità, facendosi anche loro abbindolare dal fascino dell'Hi-tech....

Quindi, come puoi ben capire, il problema di come allestire un ipotetico spazio teatrale è veramente lontano dalla realtà della situazione. 
Ma se dovessi utopicamente immaginare una situazione dove il teatro riesca a riconnettersi con il tessuto sociale lo spazio che prenderei a modello, come base di partenza, è quello che ho visto a Quebec City, in Canada, ovvero la sede di Lepage ExMachina. È una vecchia caserma dei vigili del fuoco trasformata in spazio teatrale. Naturalmente palco e platea sono modulabili e smontabili. È possibile attaccare fari, casse audio e videoproiettori ovunque. Ed ospita anche studi di creazione e registrazione video, audio e computer grafica. Quindi cercherei di avere tutte le diverse possibilità hardware e software presenti sul mercato, sia per gestire il suono, il video, le luci e le scenografie. Non voglio qui indicare precisamente cosa, anche perché penso che bisognerebbe tenersi continuamente aggiornati. Mi immagino uno spazio pubblico che non debba preoccuparsi di avere finanziamenti e che sia gestito da persone in grado di riconoscere quali sono le macchine davvero necessarie. Ma soprattutto mi preoccuperei di avere un'equipe di programmatori e ingegneri elettronici in grado di creare o modificare software, e hardware, open source da poter poi mettere a disposizione di altri teatri e artisti.

Se invece abbiamo a disposizione un piccolo spazio e pochi soldi bastano anche solo un PC per gestire il video, un videoproiettore, una videocamera, un PC per il suono e un buon impianto audio. E naturalmente qualche faro con mixer luci.

Le Telestreet potrebbero virtualmente essere ovunque, potrebbe di fatto esserci un free broadcasting di contenuti e pensiero senza transiti dentro i castelli del potere televisivo di questo paese. Altra utile provocazione: mi elenchi un palinsesto immaginario di un giorno di una web tv (ironia ammessa )?

Eccolo:

Dalle 7,00 alle 9,00

Diretta dal bar del quartiere: "cappuccini e opinioni" sul giorno che comincia, lettura dei giornali locali e libere farneticazioni dai clienti del bar.

Dalle 9,00 alle 12,00

Diretta dalla bottega: "cavoli e merende" ovvero informazioni e commenti liberi sui prezzi dei beni di consumo e comparazioni tra i diversi negozi. Pettegolezzi sui fatti del quartiere. Baratto di quello che si vuole. 
Dalle 12,00 alle 15,00

Salvaschermo di quartiere.

Chiunque può inviare in redazione immagini, o fotografie, da mandare a ciclo continuo come un salvaschermo.

Possono passare anche messaggi inviati via SMS o Internet.

Dalle 15,00 alle 20,00

Spazio libero e imprevedibile.

Trasmissione di servizi su questioni del quartiere, servizi di altre telestreet, e diretta con telefonate tra lo studio e le case su qualsiasi cosa passi per la testa e senza alcun vincolo di orario o palinsesto.

Dalle 20,00 alle 7,00

Libera camera di controllo.

Una telecamera, che inquadra un pezzo di strada o piazza, di fronte alla quale chiunque può fermarsi e dire-fare quello che vuole.

Questo palinsesto può essere stravolto e modificato in qualsiasi momento e senza preaviso.

\section{Ti ritieni un Punk delle tecnologie legate anche al teatro?}

Bisogna vedere cosa intendi per Punk. Mi sono accorto di essere stato un Cyberpunk quando ancora non ne conoscevo la "definizione" ma utilizzavo l'estetica degli "occhiali a specchio" e dell'adrenalina digitale con la "Bandamagnaetica", erano gli anni '80. Se per Punk si intende la concezione del "fatti la tua musica anche se non sai suonare" allora sì, sono un Punk. Non mi riconosco invece nell'estetica "borchie, spille, catene e tatuaggi". Utilizzare adesso il concetto Punk o Cyber-punk lo trovo sviante. Mi sento più vicino alle concezioni del "reality Hacker" dove si dice che l'attitudine Hacker si può esprimere anche in contesti che vanno oltre lo spazio virtuale dei computers. E per questo che ultimamente ho definito la storia della mia pratica teatrale "Hacker Teatro". Ma ormai è una storia passata... Cosa mi ritengo adesso lo saprò tra qualche anno... quando il presente sarà ormai passato. 


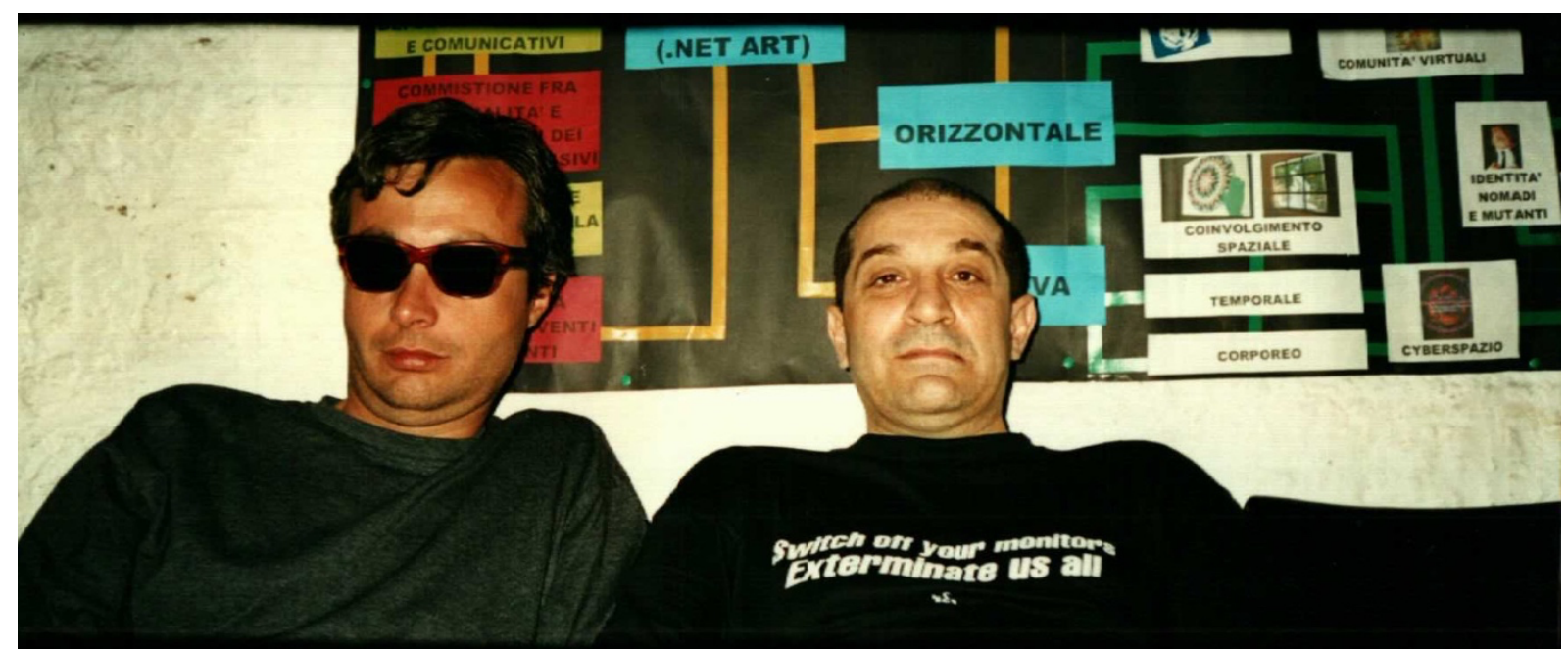

Giacomo Verde con Francesco Galluzzi ad Hackmeeting 2000.

\section{Biografia dell'autore}

Regista underground, noto anche per il cortometraggio R.A.C.H.E. che prende le fila dalle suggestioni eymerichiane di Valerio Evangelisti, nonché animatore tecnologico in AR/VR e cultore di un fantastico connesso alle strutture angolari del nostro universo casualmente antropico, dalla fine degli ' 80 , con il seminale Syrena, Mariano Equizzi investiga e interferisce nella zona incognita che si trova al confine tra tecnologie digitali e rivoluzioni creative delle avanguardie del secolo scorso, in cui è nato e da cui trae artefatti tecno-logici per la libera azione dei millennials. www.komplex.city

Contributo fuori peer review

Si ringrazia Mariano Equizzi per l'intervista inedita che trova in questa rivista la sua prima pubblicazione. 